\title{
Quantum hair of black holes out of equilibrium
}

\author{
Ram Brustein ${ }^{1, *}$ and A. J. M. Medved ${ }^{2,3, \dagger}$ \\ ${ }^{1}$ Department of Physics, Ben-Gurion University, Beer-Sheva 84105, Israel \\ ${ }^{2}$ Department of Physics \& Electronics, Rhodes University, Grahamstown 6140, South Africa \\ ${ }^{3}$ National Institute for Theoretical Physics (NITheP), Western Cape 7602, South Africa
}

(Received 2 October 2017; published 22 February 2018)

\begin{abstract}
Classically, the black hole (BH) horizon is completely opaque, hiding any clues about the state and very existence of its interior. Quantum mechanically and in equilibrium, the situation is not much different; Hawking radiation will now be emitted, but it comes out at an extremely slow rate, is thermal to a high degree of accuracy, and thus carries a minimal amount of information about the quantum state within the BH. Here, it is shown that the situation is significantly different when a quantum BH is out of equilibrium. We argue that the BH can then emit "supersized" Hawking radiation with a much larger amplitude than that emitted in equilibrium. The result is a new type of quantum hair that can reveal the state and composition of the $\mathrm{BH}$ interior to an external observer. Moreover, the frequency and amplitude of the new hair can be explained by the observer without invoking any new physical principles. The new hair decays at a parametrically slow rate in comparison to the Schwarzschild time scale and can be detected through the emission of gravitational waves (and possibly other types of waves) whenever a BH has received a large enough surge in energy to move it far away from its equilibrium state, in particular, during and immediately after a BH-merger event. The current discussion is motivated by a previous analysis, in the context of a recently proposed polymer model for the $\mathrm{BH}$ interior, that implies emissions just like those described here. We expect, however, that the new hair is a model-independent property of quantum BHs.
\end{abstract}

DOI: 10.1103/PhysRevD.97.044035

\section{INTRODUCTION}

It was not too long ago that the classical picture of the interior of a black hole (BH) was more or less accepted. However, thanks to the so-called firewall argument [1,2] (and as recently reviewed in Ref. [3]) along with its less-celebrated forerunners [4-6], a conflict between the classical description of a $\mathrm{BH}$ and the principles of quantum theory has been revealed. As such, a variety of nonclassical models of the interior have emerged as leading candidates for a successor. (See, however, Refs. [7,8].) But a consensus point of view on the correct description of the interior is still lacking, as evident from the ongoing and rather intense debate (see Ref. [9] for a summary). Some have suggested that the BH interior should, one way or another, be expelled from the accessible part of spacetime (e.g., Ref. [10]). We, on the other hand, have suggested that the $\mathrm{BH}$ interior is composed of highly excited, interacting, long, closed strings-essentially, a "ball of string" or a collapsed polymer [11]. Others have,

\footnotetext{
ramyb@bgu.ac.il

j.medved@ru.ac.za
}

Published by the American Physical Society under the terms of the Creative Commons Attribution 4.0 International license. Further distribution of this work must maintain attribution to the author(s) and the published article's title, journal citation, and DOI. Funded by SCOAP ${ }^{3}$. however, proposed entirely different compositions for the interior (e.g., Ref. [12]).

The recent detections of gravitational waves (GWs) from BH mergers [13] have elevated what was an abstract academic debate about the laws of quantum gravity to a more tangible discussion about the expected signatures in the GW data of either a nonempty or an excised interior [14,15], meaning that each new proposal about the nature of BHs will have to confront such data as they come in from subsequent merger observations. Besides the resulting GWs, there could also be data from the emission of electromagnetic waves and neutrinos, although neither has been detected so far. Until now, the data have been completely consistent with the predictions of classical general relativity (GR) $[16,17]$ (see, however, Refs. $[15,18])$, but it is too soon to reach any definitive conclusions.

We have recently analyzed some of the consequences of our proposed polymer description of the $\mathrm{BH}$ interior. In particular, it was argued in Ref. [19] (also see Ref. [20]) that GW observations could provide a means for distinguishing our model from that of a classical BH as well as from other candidate models. The idea is that the interior matter of a polymer BH, which can be effectively viewed as a fluid, will support pulsating modes in essentially the same way that a relativistic star does. These fluid modes would exist in addition to the standard spacetime modes of the exterior, and so their spectrum would then be added onto that of the 
ringdown or quasinormal modes (QNMs) of a perturbed $\mathrm{BH}$. The polymer has an outer surface that behaves just like a $\mathrm{BH}$ horizon in the limit $\hbar \rightarrow 0$ but is otherwise only partially opaque [21]. Models without such an effective horizon would likely have a spectrum that differs even more substantially from that of a classical $\mathrm{BH}$ [22].

The bottom line is that a fluidlike description of the $\mathrm{BH}$ interior gives rise to a new type of quantum hair, which is emitted with a parametrically lower frequency $\omega_{I} \sim v_{I} / R_{S}$ and a parametrically longer damping time $\tau_{I} \sim R_{S} c / v_{I}^{2}$ [19] in comparison to the QNMs of its classical counterpart. Here, $R_{S}$ is the Schwarzschild radius, $c$ is the speed of light, and $v_{I}<c$ is the velocity of sound for a fluid mode from the Ith class. For the polymer model in particular, the parametric difference is due to the introduction of a new scale, the string scale, and therefore a new dimensionless parameter $g_{s}=l_{P} / l_{s}$, the ratio of the Planck scale to the string scale. In this case, $v_{I} / c=g_{s}$ for what would be the most experimentally accessible class of modes.

Here, we would like to discuss how a coupling between internal fluid modes and emitted GWs (or other types of waves) can occur from the perspective of an observer on the outside. The external observer does have the prerogative of ignoring all knowledge about the interior but, then again, should be able to explain all phenomena in the framework of GR. Hawking has made this same point in Ref. [23]; to wit, "All data on a 'hidden' surface compatible with the observer's limited information are equally probable." From this perspective, the interior of the $\mathrm{BH}$ is an imaginary construction of which the sole justification is to serve as a mental crutch to help explain the properties of the emitted radiation. After all, the $\mathrm{BH}$ horizon is supposed to prevent just such an emission as the interior is causally disconnected as far as this outside observer is concerned [3].

Since the picture does seem sensible enough from an internal point of view $[19,20]$, what needs to be shown is that an external observer will attribute the source of the additional (fluid) modes to perturbations of the exterior spacetime and not those of the $\mathrm{BH}$ interior. Establishing this to be true is the primary objective of the current paper, and we are indeed able to confirm that the two perspectives are consistent.

The key to resolving the conflicting viewpoints is the realization that this external perspective for the fluid modes is really no more or less paradoxical than that of Hawking radiation itself [23,24]. In spite of some arguments that the Hawking effect can be linked to mechanisms like pair production, quantum tunneling, and so on, one can only learn about the interior indirectly by observations on the outside. And so, as the above quotation correctly implies, any explanation of the Hawking process is just as viable as any other as long as its predictions are consistent with what is known or could be known about BHs. Hence, there has to be a vantage point for which the Hawking modes originate in the exterior spacetime because, just like for any other form of matter, this radiation is not permitted to escape through the horizon.
What we will then argue is that, when viewed externally, the fluid modes are describing "supersized" Hawking emissions. This is because each such event represents a largeamplitude coherent state of photons, gravitons, etc. (akin to an electromagnetic or gravitational field) rather than a single boson. And, the same as for the standard case, the supersized modes must appear to have originated in the exterior spacetime. For either choice, regular or supersized, we will assert that this exterior picture is consistent as long as the $\mathrm{BH}$ has, to some degree, deviated from its equilibrium state. The degree of deviation depends on the amount of energy that is injected into the specific mode and, therefore, also determines the amplitude of the respective emission.

The emission of supersized Hawking modes is not a regular occurrence as such a large-amplitude emission necessitates a large injection of energy. Unless the $\mathrm{BH}$ is disturbed well away from its equilibrium state, the emission of supersized radiation is highly suppressed. Indeed, the equilibrium rate of spontaneous particle production near the horizon of a $\mathrm{BH}$ can be estimated by assuming a Schwinger-like process [25]. The outcome is a rate $\mathcal{R}$ which is strongly peaked for particles of which the energy $\epsilon$ is close to that of a standard Hawking emission, $\mathcal{R} \sim e^{-\frac{2 \pi R_{S} \epsilon}{\hbar}}$. It follows that, when the BHs are quiescent, the emission of supersized Hawking radiation is an exponentially rare event. Nonetheless, supersized particle production could result from the collision of two BHs in a binary system because, in this case, the surge in energy would typically be a finite fraction of the final $\mathrm{BH}$ mass. We conclude that the GWs emitted from a BH-merger event are not only our best opportunity for observing supersized Hawking radiation but could well be our only opportunity.

We will also address the puzzling absence of (damped) relativistic modes in the interior, which was a central finding in Ref. [19]. The question of interest is whether this result is an artifact of our particular model or a physical consequence of a more general nature. We do find that this is indeed a general phenomenon, from both the internal and external perspectives.

Although relying on the results of a particular model, we expect that many of the ideas and conclusions should apply just as well to any "BH-like" object; which is meant as an exotic spacetime containing exotic matter that can exist inside of an ultracompact object while somehow resisting gravitational collapse. This object should, simultaneously, exhibit all of the standard properties of a $\mathrm{BH}$ when viewed from the outside. Note that this excludes models lacking a "horizonlike" outer surface such as gravastars and wormholes.

The above claims are substantiated and further discussed in Sec. II, followed by a summary in Sec. III. Throughout the paper, we ignore numerical factors of order 1 , and fundamental constants are only made explicit when needed for clarity. For simplicity, three large spacelike dimensions and a nonrotating $\mathrm{BH}$ are assumed. When we refer to $\mathrm{BHs}$ 
(including the use of the subscript $B H$ ), the polymer model is implied unless stated otherwise. As the current results are often compared to those in Ref. [19], we follow this earlier treatment and assume that the fluid modes are scalars.

Before proceeding, let us be more careful about our use of the term "hair" in the current context. As is well known, the only hair which BHs in equilibrium can have is associated with gauge fields and their conserved charges. It is also well understood that, when a BH is perturbed, it will return to its equilibrium state through the shedding of additional hair which is not associated with any gauge symmetries. This process of shedding can be expected to be exponential in some time scale. Our basic claim is that, when the BHs are not those of GR, there could well be another time scale, in addition to the Schwarzschild or light-crossing time, which is involved in this hair-shredding process. But, even after a short period of shedding, a $\mathrm{BH}$ would look almost exactly like one of GR of which the hair is indeed associated with symmetries.

\section{EXTERNAL PERSPECTIVE}

An external observer can, from her perspective, only see "stuff" which is on her side of the horizon. Whatever is supposed to be leaking out of the $\mathrm{BH}$, whether it be conventional or supersized Hawking radiation, must have originated from outside the horizon as far as this observer is concerned. When the $\mathrm{BH}$ is close to its equilibrium state, this outside observer can use a "horizon-locking gauge" to describe the near-horizon geometry [26,27]. In this gauge, the equilibrium position of the compact object's outer surface (or effective horizon) stays at $r=R_{S}$ up to some high order in the relative strength of the perturbation.

To understand how an outside observer would interpret the supersized quantum radiation, it is necessary to know about departures from equilibrium. To get a handle on this, let us first recall a classical analog: a tidal deformation of the horizon of a slowly rotating $\mathrm{BH}$ due to an external perturbation. This was first discussed by Hartle [28] and later by O'Sullivan and Hughes [29] (see, in particular, Appendix B1 of Ref. [29] and also Refs. [26,27]), who visualized this setup by embedding a deformed sphere in a three-dimensional, flat Euclidean space. The basic idea was to lock up the position of the sphere's outer surface (as described above) and rather interpret its deformation as a perturbation in the associated Ricci curvature. But one can just as well choose a gauge for which the deformation is interpreted as the difference between the location of the outer surface and $R_{S}$. This difference would, in our case, be the extent to which the internal fluid is either protruding out of or sinking into the fiducial horizon.

Figure 1 can help one visualize the gravitational coupling between the deformed horizon and the external observer. For example, a static quadrupole deformation of the horizon (rightmost panel, Fig. 1) changes the sphere from its unperturbed shape by an amount that scales with the strength of the perturbation times the second Legendre polynomial for the polar angle $P_{2}(\theta) \propto 3 \cos ^{2} \theta-1$. More generally, the position and shape of the deformed horizon can be expected to oscillate in time.

Perhaps contrary to expectations, the deformed surface can include both depressions and protrusions irrespective of the direction of the perturbing force, as illustrated in Fig. 1. Our interest is in places where the horizon is depressed inward (equivalently, where the fluid protrudes outward), as this implies that a portion of the interior has been momentarily "exposed" to the exterior spacetime. From an external point of view, such a depression of the horizon can only be explained by the $\mathrm{BH}$ absorbing a flux of negative energy, just as the emission of standard Hawking radiation is normally explained [30]. However, the negative flux is really just a story that an outside observer has to invent to reconcile energy conservation with the flux of positive energy emanating out from the $\mathrm{BH}$.

In the classical case of tidal horizon deformations, an outgoing flux occurs only for the superradiant modes of a

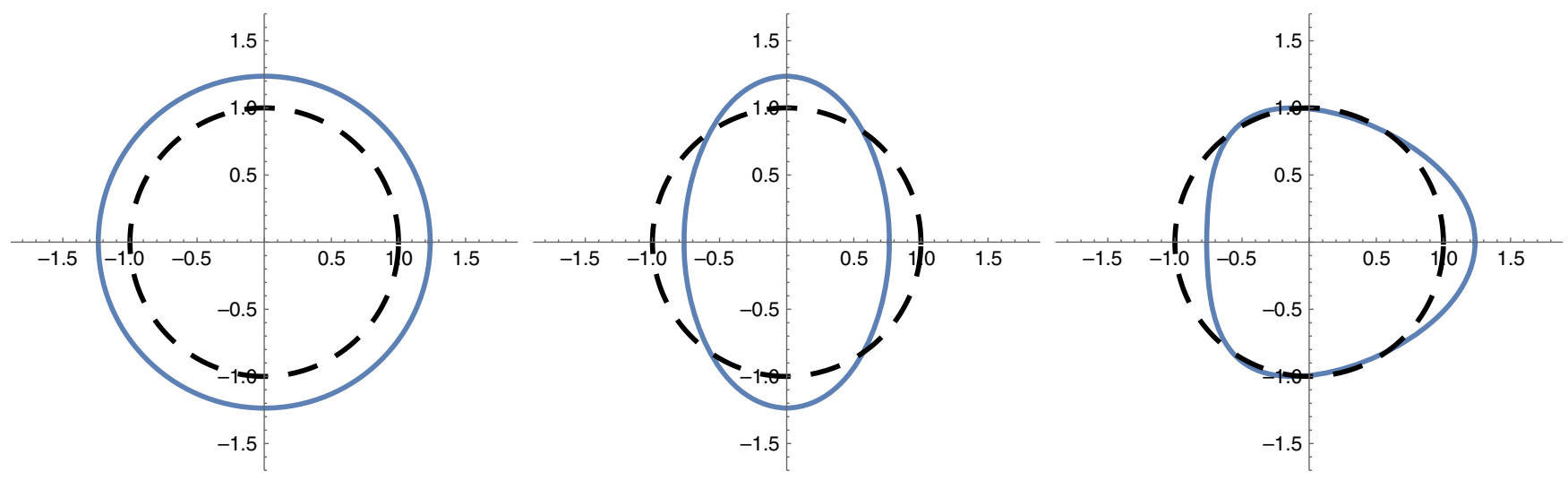

FIG. 1. Visualization of the deformed horizon. Scalar (left), dipole (center), and quadrupole (right) deformations are shown. The dashed, black circles depict the position of the unperturbed horizon at $r=R_{S}$, and the solid, blue shapes depict its respective deformations. 
rotating $\mathrm{BH}$. In the case of Hawking radiation emerging from a polymer $\mathrm{BH}$, the outgoing flux and compensating negative flux are explained, internally, by a quantum effect that allows small loops of string to break off and detach from the stringfilled interior. Supersized Hawking radiation should be similar, but, in this case, a large portion of string would be detached collectively in a short span of time.

Also from an internal perspective, the relative deformation $\Delta L / L$ of the horizon due to a particular restoring force (say the $I$ th one) is the ratio ${ }^{1}$

$$
\left.\frac{\Delta L}{L}\right|_{I} \sim \frac{(\Delta E)_{I}}{E}=\frac{p_{I}}{\rho}=\frac{v_{I}^{2}}{c^{2}},
$$

where $v_{I}$ is again the sound velocity for the $I$ th mode, $p_{I}$ is its pressure, and $\rho \sim M_{\mathrm{BH}} / R_{S}^{3}$ is the total energy density ( $M_{\mathrm{BH}}$ is the $\mathrm{BH}$ mass). Here, we have employed standard relations from thermodynamics, between stress and strain and between the pressure-to-energy-density ratio and sound velocity.

Equation (1) can be used to obtain an expression for the redshift at the outermost extent of the protruding fluid,

$$
\left.\sqrt{-g_{t t}}\right|_{I}=\sqrt{1-\frac{R_{S}}{r_{I}}}=\sqrt{1-\frac{R_{S}}{R_{S}\left(1+\left.\frac{\Delta L}{L}\right|_{I}\right)}} \approx \sqrt{\left.\frac{\Delta L}{L}\right|_{I}},
$$

that is,

$$
\left.\sqrt{-g_{t t}}\right|_{I} \approx v_{I} / c .
$$

The above estimates will be used to determine the mode frequencies as measured by an observer in the exterior, which will tell us if her observations are consistent with those from an interior point of view.

For future reference, it should be noted that Eqs. (2) and (3) are not compatible with a relativistic speed of sound $v_{I}=c$ since, in this case, $\Delta L / L$ cannot be small. This is the first indication that the limiting case of $v_{I}=c$ is problematic.

\section{A. Hawking radiation}

To illustrate the procedure of determining the external frequencies, it is useful to start with the familiar case of standard Hawking radiation. Let us then begin with

$$
(\Delta E)_{H} \sim T_{H}=\frac{1}{R_{S}}
$$

and

\footnotetext{
${ }^{1}$ Unlike in Ref. [19], we now use the energy $E$ in place of the free energy $F$, as these and their order-by-order corrections scale in parametrically the same way in the polymer model.
}

$$
E=M_{\mathrm{BH}}
$$

from which it follows that

$$
\frac{(\Delta E)_{H}}{E} \sim \frac{1}{R_{S} M_{\mathrm{BH}}}=\frac{1}{S_{\mathrm{BH}}}
$$

and then

$$
\left.\sqrt{-g_{t t}}\right|_{H}=\sqrt{\frac{1}{S_{\mathrm{BH}}}}=\frac{l_{P}}{R_{S}},
$$

where a subscript of $H$ indicates an associated property (e.g., $T_{H}$ is the Hawking temperature), $S_{\mathrm{BH}}$ is the $\mathrm{BH}$ entropy, and $l_{P}$ is the Planck length.

This is the redshift at the location of the protruding fluid and, therefore, the location of the source as far as an external observer is concerned. But what frequency would this observer assign to a Hawking mode at the same point? It is natural to ascribe a wavelength of $l_{P}$ to a near-horizon Hawking mode. The logic here is that, for a $\mathrm{BH}$ in equilibrium, there can only be two length scales: namely, the Schwarzschild radius and the Planck length. A state of equilibrium must be the case (to very good approximation) for the emission of standard Hawking radiation, as a macroscopically large $\mathrm{BH}$ emits radiation at a very slow rate and the energy of each emission is a tiny fraction of the total mass.

And so the external observer assigns a Hawking mode with a frequency at the source of $\omega_{\text {source }}^{(H)}=c / l_{P}$. The frequency at the location of the external observer is then found by redshifting its value at the source. This process yields the expected result,

$$
\omega_{\text {ext }}^{(H)}=\left.\omega_{\text {source }}^{(H)} \sqrt{-g_{t t}}\right|_{H}=T_{H} .
$$

This is not exactly ground-breaking physics, as the above argument runs along the same lines as that of the membrane paradigm [31]. The difference here, though, is that we did not have to conjecture a location for the (so-called) stretched horizon before determining the redshift.

The polymer model realizes the same value for the Hawking temperature from an internal perspective [21]. A small loop of string which has broken off from one of the typically long loops in what is a bound state of interacting, highly excited, closed strings will have some probability of escape, and a calculation reveals that both the rate and energy of emission agree with $T_{H}$. In this way, consistency between the exterior and interior perspectives has been established, at least as far as it concerns the case of conventional Hawking radiation. 


\section{B. Supersized Hawking radiation}

\section{Frequency of emission}

Let us next consider some nonrelativistic fluid mode, beginning with its frequency as seen by an external observer. We already know that the redshift at the location of the protruding fluid (which is exterior to but still in the vicinity of $r=R_{S}$ ) is $v_{I} / c$, and so it becomes prudent to ask about the mode frequency at this same "source" location. An external observer, who is unaware of the fluid, would confuse these nonrelativistic fluid modes with relativistic spacetime QNMs, for which the wavelength at the source would be approximately $R_{S}$. She would therefore assign them a frequency at the source of

$$
\omega_{\text {source }}^{(I)}=\frac{c}{R_{S}},
$$

from which one can deduce that

$$
\omega_{\mathrm{ext}}^{(I)}=\left.\omega_{\mathrm{source}}^{(I)} \sqrt{-g_{t t}}\right|_{I}=\frac{v_{I}}{R_{S}}
$$

again as expected (see the Introduction). The difference between the internal and external perspectives can then only be one of interpretation.

Since the frequencies redshift, one might wonder why the energies $(\Delta E)_{I}$ in Eq. (1) do not. In reality they do, but, in both cases (standard and supersized), the values that were used for $(\Delta E)_{I}$ and $E$ are already what would be measured by an asymptotic observer. We know this because the standard Hawking case can be used to calibrate all other cases. The two energies $(\Delta E)_{I}$ and $E$ at the source would then be blueshifted from their asymptotic values in the same way, leaving their ratio undisturbed.

\section{Coupling to the exterior and decay time}

To complete our consistency check, the estimates from Ref. [19] for an emitted energy of $(\Delta E)_{I}=v_{I}^{2} E_{I}$ and a damping time of $\tau_{I}=R_{S} / v_{I}^{2}$ need to be similarly reproduced from an external perspective. Here, $E_{I}$ is the amount of energy which has been injected into the Ith mode by the deforming force. (It was assumed in Ref. [19] that $\left.E_{I} \sim M_{\mathrm{BH}}.\right)$

For an exterior observer, the supersized Hawking radiation is relativistic and has a frequency of $\omega_{I}=v_{I} / R_{S}$. Therefore, she must conclude that the wavelength of the radiation at distances far away from the horizon is $\lambda_{I}=R_{S} c / v_{I}$. The same conclusion can be arrived at by the fact that, like before, the wavelength near the source must be $\lambda \sim R_{S}$, which then asymptotically redshifts to $\lambda_{I} \sim R_{S} c / v_{I}$.

Now, consider that the same observer attributes the source with a radial size of about $R_{S}$. She then just needs to know that the transmission cross section for such long wavelength modes through a proportionally smaller surface of area $A$ is determined by the ratio $A / \lambda^{2}$, which translates into $R_{S}^{2} / \lambda_{I}^{2}=v_{I}^{2}$ for the case at hand. We can conclude that the coupling or efficiency of emission goes as $v_{I}^{2}$, so that the energy in the emitted wave scales as

$$
(\Delta E)_{I} \sim E_{I} v_{I}^{2},
$$

in agreement with the internal perspective [19]. This is based on the assumption that most of the mode energy is being emitted in the form of coherent waves rather than dissipating as heat.

Meanwhile, the damping time for any given mode $\tau_{I}$ is directly related to the corresponding relaxation time of the $\mathrm{BH}$. The latter can be deduced with an inspection of

$$
\frac{d E_{I}}{d t} \propto(\Delta E)_{I} \sim v_{I}^{2} E_{I},
$$

which implies a relaxation time that scales with the inverse of $v_{I}^{2}$ and likewise for the damping time $\tau_{I}$. One is then led to the expected result of $\tau_{I} \sim R_{S} / v_{I}^{2}$, where the factor of $R_{S}$ follows simply for dimensional reasons and the knowledge that the Schwarzschild time is the only classically available time scale.

In summary, the supersized Hawking radiation oscillates with a frequency of $\omega_{I}=v_{I} / R_{S}$, carries away an energy of $(\Delta E)_{I}=E_{I} v_{I}^{2}$ (where it is expected that $E_{I} \sim M_{\mathrm{BH}}$ ), and decays with a characteristic time of $\tau_{I}=R_{S} / v_{I}^{2}$. This can be compared to the standard Hawking emissions with a frequency of $\omega_{H}=1 / R_{S}$, an effective coupling of $v_{H}^{2}=1$ (cf. $\omega_{I}=v_{I} / R_{S}$ ), and an emitted energy of $1 / R_{S}$, leading to a decay time of $\tau_{H}=R_{S} / v_{H}^{2}=R_{S}$, again just as expected.

\section{Coupling to gravitational waves}

Also of interest is the strength of the coupling of the fluid modes to external GWs, as this along with $(\Delta E)_{I}$ is what determines the amplitude of the emitted GWs. The coupling strength can be determined using Einstein's quadrupole formula

$$
\langle h\rangle \sim \frac{1}{r} \ddot{Q} .
$$

This means that, for an external observer,

$$
\langle h\rangle_{I} \sim \ddot{Q}_{I} \sim(\Delta E)_{I} R_{S}^{2} \omega_{I}^{2} \sim\left(E_{I} v_{I}^{2}\right)\left(R_{S} \omega_{I}\right)^{2} \sim E_{I} v_{I}^{4},
$$

where the factor of $R_{S}^{2}$ can be attributed to the quadrupole moment of the emitting object and a dot denotes a time derivative. Also, the two factors of frequency are due to the pair of time derivatives, which further suppresses the amplitude of the emitted GWs. Equation (14) agrees with the internal version of the same calculation [19]. 


\section{Absence of relativistic modes}

From an internal perspective, the absence of relativistic fluid modes can be traced to the polymer being near its equilibrium state and an incompatibility between the two boundary conditions that any fluid mode is required to satisfy: vanishing at the center of the object and outgoing at its surface. Moreover, the leading correction to the (free) energy has to be parametrically small, $\Delta E / E<1$ [see Eq. (1) and the comment just before Sec. II A], from which it follows that $v_{I}^{2} / c^{2}=\Delta E / E<1$.

From an external perspective, it is rather the continuity of the emission at $v_{I}=1$ which makes the emission of such waves impossible. This is because $v_{I}>1$ is unphysical and therefore unacceptable; the amplitude of such faster-thanlight waves has to vanish identically. The condition of continuity then implies that the amplitude of waves for which $v_{I}=1$ must similarly vanish.

Let us explain the continuity argument in a more detailed way: An external relativistic mode could never have been redshifted, as this would imply that it had been sourced by a fluid mode of which the sound velocity was faster than the speed of light. Now, consider that, for a (would-be) relativistic fluid mode, $\omega=\alpha c / R_{S}$ and $\lambda=R_{S} / \alpha$, where $\alpha$ is some constant of order 1 which takes into account any neglected numerical factors. But if its wavelength is indeed $R_{S} / \alpha \simeq R_{S}$, this mode must have originated somewhere close to the horizon and must then have experienced a significant redshift. Conversely, to suffer no redshift, it would have to be produced far away from the horizon with a wavelength that is parametrically larger than $R_{S}$. Such a mode could not possibly be under the influence of the $\mathrm{BH}$ and so-even if it somehow defied the condition of continuity and did exist-an external observer would not consider it to be part of the BH's QNM spectrum. This argument does not preclude the existence of the standard class of relativistic spacetime QNMs, as these are a consequence of waves in the exterior spacetime and not of fluid modes from inside the $\mathrm{BH}$.

\section{Potential barrier}

Finally, we would now like to show that the gravitational potential barrier at about $\frac{3}{2} R_{S}$ does not affect in any significant way the emission of the supersized Hawking radiation; an assumption that was implicit in Ref. [19].

To understand this claim, let us consider a massless particle with a modest angular momentum; then, the peak in the barrier goes as $1 / R_{S}$ when expressed in units of energy (rather than units of energy squared as it normally appears). That the peak is of the same order as $T_{H}$ is what explains the famous graybody factors affecting the emission of the standard Hawking radiation (e.g., Ref. [9]). On the other hand, the energy of a supersized emission is of order $M_{\mathrm{BH}} v_{I}^{2}$ for scalar modes and $M_{\mathrm{BH}} v_{I}^{4}$ for gravitons, as
$E_{I} \sim M_{\mathrm{BH}}$ can be expected, meaning that the ratio of the radiated energy to the height of the barrier is $M_{\mathrm{BH}} v_{I}^{4} /\left(1 / R_{S}\right)=S_{\mathrm{BH}} v_{I}^{4} \gg 1$ for the fluid modes of interest (i.e., those for which the resulting GWs could be experimentally detected). A supersized emission, which is really a large coherent state of gravitons, will not be affected by the barrier at all. This has become a classical problem in which the energy of the wave far exceeds that of the potential barrier.

\section{CONCLUSION}

It was shown that, if an ultracompact object is nonempty but does have a surface that acts effectively as a $\mathrm{BH}$ horizon, interior modes can nevertheless couple to emitted GWs or, for that matter, other types of waves (such as electromagnetic waves and neutrinos). An external observer will view the interior modes as supersized Hawking emissions which originated close to but outside the equilibrium position of the horizon. Moreover, we have shown that the same point of view applies just as well to standard Hawking radiation.

Although these conclusions rely on the intuition gained from studying the polymer model for the $\mathrm{BH}$ interior [19] (also Ref. [20]), we believe that they are not specific to the polymer model and would readily carry over to any ultracompact object containing nontrivial fluidlike matter and having an outer surface that acts like a $\mathrm{BH}$ horizon to some level of approximation.

The resulting picture is suggestive of a new type of $\mathrm{BH}$ hair for which a parametrically long time of shedding is required. In fact, the existence of novel $\mathrm{BH}$ hair should be part and parcel for any BH-like object containing nontrivial matter and could yet be the key which unlocks the door to the secretive world behind the horizon. Such revelations could come about through the observation of GWs resulting from $\mathrm{BH}$ mergers, hopefully in the near future.

We have also addressed the absence of damped relativistic modes in the interior, even though these are ubiquitous in some of the analogous calculations for relativistic stars. There is, however, some evidence that the suppression of relativistic fluid modes is a more general phenomenon (e.g., Refs. [32,33]). If so, our analysis could prove helpful in a broader range of studies.

\section{ACKNOWLEDGMENTS}

The research of R. B. was supported by the Israel Science Foundation Grant No. 1294/16. The research of A. J. M. M. received support from NRF Incentive Funding Grant No. 85353 and NRF Competitive Programme Grant No. 93595. A. J. M. M. thanks Ben Gurion University for their hospitality during his visit. 
[1] A. Almheiri, D. Marolf, J. Polchinski, and J. Sully, Black Holes: Complementarity or Firewalls?, J. High Energy Phys. 02 (2013) 062.

[2] D. Marolf and J. Polchinski, Gauge/Gravity Duality and the Black Hole Interior, Phys. Rev. Lett. 111, 171301 (2013).

[3] S. D. Mathur, Resolving the black hole causality paradox, arXiv:1703.03042.

[4] N. Itzhaki, Is the Black Hole Complementarity principle really necessary?, arXiv:hep-th/9607028.

[5] S. D. Mathur, What exactly is the information paradox?, Lect. Notes Phys. 769, 3 (2009).

[6] S. L. Braunstein, S. Pirandola, and K. Zyczkowski, Entangled Black Holes as Ciphers of Hidden Information, Phys. Rev. Lett. 110, 101301 (2013).

[7] S. Raju, Smooth causal patches for AdS black holes, Phys. Rev. D 95, 126002 (2017).

[8] R. van Breukelen and K. Papadodimas, Quantum teleportation through time-shifted AdS wormholes, arXiv:1708.09370.

[9] D. Harlow, Jerusalem lectures on black holes and quantum information, Rev. Mod. Phys. 88, 015002 (2016).

[10] G. 't Hooft, The firewall transformation for black holes and some of its implications, Found. Phys. 47, 1503 (2017).

[11] R. Brustein and A. J. M. Medved, Black holes as collapsed polymers, Fortschr. Phys. 65, 1600114 (2017).

[12] G. Dvali and C. Gomez, Black hole's quantum N-portrait, Fortschr. Phys. 61, 742 (2013).

[13] B. P. Abbott et al. (LIGO Scientific and Virgo Collaborations), Observation of Gravitational Waves from a Binary Black Hole Merger, Phys. Rev. Lett. 116, 061102 (2016); Binary Black Hole Mergers in the First Advanced LIGO Observing Run, Phys. Rev. X 6, 041015 (2016); GW170104: Observation of a 50-Solar-Mass Binary Black Hole Coalescence at Redshift 0.2, Phys. Rev. Lett. 118, 221101 (2017).

[14] C. F. B. Macedo, V. Cardoso, L. C. B. Crispino, and P. Pani, Quasinormal modes of relativistic stars and interacting fields, Phys. Rev. D 93, 064053 (2016).

[15] J. Abedi, H. Dykaar, and N. Afshordi, Echoes from the Abyss: Evidence for Planck-scale structure at black hole horizons, Phys. Rev. D 96, 082004 (2017).

[16] N. Yunes, K. Yagi, and F. Pretorius, Theoretical physics implications of the binary black-hole mergers GW150914 and GW151226, Phys. Rev. D 94, 084002 (2016).

[17] B. P. Abbott et al. (LIGO Scientific and Virgo Collaborations), Tests of General Relativity with GW150914, Phys. Rev. Lett. 116, 221101 (2016).

[18] G. Ashton et al., Comments on: "Echoes from the abyss: Evidence for Planck-scale structure at black hole horizons", arXiv:1612.05625.
[19] R. Brustein, A. J. M. Medved, and K. Yagi, When black holes collide: Probing the interior composition by the spectrum of ringdown modes and emitted gravitational waves, Phys. Rev. D 96, 064033 (2017).

[20] R. Brustein, A. J. M. Medved, and K. Yagi, Discovering the interior of black holes, Phys. Rev. D 96, 124021 (2017).

[21] R. Brustein and A. J. M. Medved, Emergent horizon, Hawking radiation and chaos in the collapsed polymer model of a black hole, Fortschr. Phys. 65, 1600116 (2017).

[22] V. Cardoso and P. Pani, The observational evidence for horizons: from echoes to precision gravitational-wave physics, arXiv:1707.03021; Tests for the existence of horizons through gravitational wave echoes, Nat. Astron. 1, 586 (2017).

[23] S. W. Hawking, Breakdown of predictability in gravitational collapse, Phys. Rev. D 14, 2460 (1976).

[24] S. W. Hawking, Black hole explosions, Nature (London) 248, 30 (1974); Particle creation by black holes, Commun. Math. Phys. 43, 199 (1975).

[25] R. Brustein and A. J. M. Medved, Constraints on the quantum state of pairs produced by semiclassical black holes, J. High Energy Phys. 07 (2015) 012.

[26] E. Poisson and I. Vlasov, Geometry and dynamics of a tidally deformed black hole, Phys. Rev. D 81, 024029 (2010).

[27] I. Vega, E. Poisson, and R. Massey, Intrinsic and extrinsic geometries of a tidally deformed black hole, Classical Quantum Gravity 28, 175006 (2011).

[28] J. B. Hartle, Tidal friction in slowly rotating black holes, Phys. Rev. D 8, 1010 (1973).

[29] S. O'Sullivan and S. A. Hughes, Strong-field tidal distortions of rotating black holes: Formalism and results for circular, equatorial orbits, Phys. Rev. D 90, 124039 (2014); Erratum, Phys. Rev. D 91, 109901 (2015).

[30] N. D. Birrell and P.C.W. Davies, Quantum Fields in Curved Space (Cambridge University Press, Cambridge, England, 1982).

[31] K. Thorne, R. Price, and D. MacDonald, Black Holes: The Membrane Paradigm (Yale University, New Haven, CT, 1986).

[32] N. Andersson, K. D. Kokkotas, and B. F. Schutz, Spacetime modes of relativistic stars, Mon. Not. R. Astron. Soc. 280, 1230 (1996).

[33] G. Allen, N. Andersson, K. D. Kokkotas, and B. F. Schutz, Gravitational waves from pulsating stars: Evolving the perturbation equations for a relativistic star, Phys. Rev. D 58, 124012 (1998). 